\title{
CORRECTION
}

\section{Author Correction: Skp2 dictates cell cycle-dependent metabolic oscillation between glycolysis and TCA cycle}

Jing Liu (D), Yunhua Peng, Le Shi, Lixin Wan (D), Hiroyuki Inuzuka, Jiangang Long, Jianping Guo, Jinfang Zhang (D), Min Yuan, Shuangxi Zhang, Xun Wang (D, Jing Gao, Xiangpeng Dai, Shozo Furumoto, Lijun Jia, Pier Paolo Pandolfi (D), John M. Asara, William G. Kaelin Jr., Jiankang Liu and Wenyi Wei ii

Cell Research (2021) 31:104; https://doi.org/10.1038/s41422-020-00406-4

Correction to: Cell Research https://doi.org/10.1038/s41422-0200372-z, published online 15 July 2020

The author affiliations were mixed up in the previous published version. In addition, the author contribution section missed a statement of "The idea was conceived by J.K.L., W.W. and J.L.". This correction does not affect the description of the results or the conclusion of this work. We apologize for any inconvenience this may have caused.

The corrected Author affiliations, and Author contribution are as follows:

Jing Liu ${ }^{1,2}$, Yunhua Peng ${ }^{1}$, Le Shi ${ }^{1}$, Lixin Wan ${ }^{2,3}$, Hiroyuki Inuzuka ${ }^{2}$, Jiangang Long ${ }^{1}$, Jianping Guo ${ }^{2,4}$, Jinfang Zhang ${ }^{2,5}$, Min Yuan ${ }^{6}$, Shuangxi Zhang ${ }^{1}$, Xun Wang ${ }^{1,7}$, Jing Gao ${ }^{1}$, Xiangpeng Dai $^{2}$, Shozo Furumoto ${ }^{8}$, Lijun Jia ${ }^{9}$, Pier Paolo Pandolf ${ }^{10}{ }^{1}$, John M. Asara ${ }^{6}$, William G. Kaelin Jr. ${ }^{11,12}$, Jiankang Liu ${ }^{1}$ and Wenyi Wei ${ }^{2}$

${ }^{1}$ Center for Mitochondrial Biology and Medicine, The Key Laboratory of Biomedical Information Engineering of Ministry of Education, School of Life Science and Technology and Frontier Institute of Science and Technology, Xi'an Jiaotong University, Xi'an, Shaanxi 710049, China; ${ }^{2}$ Department of Pathology, Beth Israel Deaconess Medical Center, Harvard Medical School, Boston, MA 02215, USA; ${ }^{3}$ Department of Molecular Oncology, H. Lee Moffitt Cancer Center and Research Institute, Tampa, FL 33612, USA; ${ }^{4}$ Institute of Precision Medicine, the First Affiliated Hospital,
Sun Yat-sen University, Guangzhou, Guangdong 510275, China; ${ }^{5}$ Medical Research Institute, School of Medicine, Wuhan University, Wuhan, Hubei 430071, China; ${ }^{6}$ Division of Signal Transduction, Beth Israel Deaconess Medical Center and Department of Medicine, Harvard Medical School, Boston, MA 02215, USA; ${ }^{7}$ Children's Medical Center Research Institute, University of Texas Southwestern Medical Center, Dallas, TX 75390, USA; ${ }^{8}$ Division of Radiopharmaceutical Chemistry, Cyclotron and Radioisotope Center, Tohoku University, Sendai 980-8578, Japan; ${ }^{9}$ Cancer Institute, Longhua Hospital, Shanghai University of Traditional Chinese Medicine, Shanghai 200032, China; ${ }^{10}$ Cancer Research Institute, Beth Israel Deaconess Cancer Center, Department of Medicine and Pathology, Beth Israel Deaconess Medical Center, Harvard Medical School, Boston, MA 02215, USA; ${ }^{11}$ Howard Hughes Medical Institute, Chevy Chase, MD, USA; ${ }^{12}$ Department of Medical Oncology, Dana-Farber Cancer Institute, Harvard Medical School, Boston, MA, USA.

\section{AUTHOR CONTRIBUTIONS}

The idea was conceived by J.K.L., W.W. and J.L., J.L. and Y.P. designed and performed most of the experiments with assistance from L.S., L.W., H.I., J.G.L., J.P.G., J.Z., S.Z., X.W., J.G., X.D., S.F. and L.J., M.Y. and J.M.A. performed the LC-MS/MS metabolomic profiling and mass spectrometry analysis of IDH1 T157 phosphorylation. W.W., J.K.L., P.P.P. and W.G.K. supervised the study. J.L. and W.W. wrote the manuscript. All authors commented on the manuscript. 